\title{
Detection of the movement direction by the cells with directional receptive fields in the primary visual cortex of the cat
}

\author{
Ausra Daugirdiene $^{1 *}$, Algimantas Svegzda ${ }^{2}$, Romualdas Satinskas $^{2}$, Henrikas Vaitkevicius $^{2}$ \\ ${ }^{1}$ Department Psychology Didactics, Vilnius Pedagogical University, Vilnius, Lithuania; *Corresponding Author: \\ ausra.daugirdiene@gmail.com \\ ${ }^{2}$ Department of General Psychology Vilnius University, Vilnius, Lithuania
}

Received 24 July 2010; revised 2 August 2010; accepted 11 August 2010.

\begin{abstract}
The study was performed on neurons with direction selective (DS) receptive fields (RFs) in the primary visual cortex of the cat. Preferred directions (PDs) of these cells to a single light spot and a system of two identical light spots moving across the RF with a given angle between them were compared. Directional interactions appeared when the angles between the directions of the two moving spots were $30^{\circ}$ or $60^{\circ}$. PD for $56 \%$ of the cells coincided with bisectors of these angles. These cells responded to a combination of the two moving stimuli as if only one stimulus moved in the RF in an intermediate direction. This direction coincided with PD of the DS neuron to a single spot. Also, the investigation revealed that DS neurons responded to stimuli moving at such angles as $180^{\circ}$ (to preferred and opposite directions simultaneously). In the further experiment we investigated responses of the DS cells in the primary visual cortex of RF. The angle between the directions of the two moving spots was $60^{\circ}$. These cells responded to a combination of the two moving stimuli as if only one stimulus moved in RF in an intermediate direction. The more relative luminance of one of spots in pair was, the closer the intermediate direction approached to the direction of this spot).
\end{abstract}

Keywords: Cat; Primary Visual Cortex; Directionally Selective cells; Receptive Field (RF)

\section{INTRODUCTION}

The perception of the motion of an object deals with the detection of the movement direction. A retina is the firs stage where DC cells are discovered in the cat's visual system $[1,2]$. The DS cells respond preferentially to a stimulus moving in a particular direction (preferably to these cells). DS cells of the primary visual cortex of the cat do not have any direct projection from retina via the dorsal lateral geniculate nucleus (LGNd) [3-5]. This suggests that DS is mainly generated within the primary visual cortex.

It is known that area 17 and 18 have parallel input from LGNd [6], have same types of cells receptive fields (RFs) $[7,8]$ and may be considered as a primary visual cortex [9]. The cortical mechanism of direction selectivity is under constant interest $[8,10,11]$. The employment of various types of stimuli reveals the specific features of selectivity in RFs of DS cortical neurons [12-14], which can be useful in clarifying the basis of DS.

The first aim of the study was to investigate how the PDs of DS cells depend on the interaction, which two stimuli evoke moving in different directions across the RF.

It is known that there are neurons selective to direction of the stimulus motion in the visual system that, as is believed, is related with the mechanisms of the movement analysis $[15,16]$. The properties of this directional interaction in RF of DS neurons were the aim of the further study. We suggested elucidating them by exploring two moving light spots with different relative luminance contrast. We investigated how the PD of DS cells depends on the relative luminance of two light spots moving in different directions across the RF.

\section{METHODS}

Influence of the angular separation between stimuli. The investigation was performed on twenty adult cats (weight 2.5-3.5 kg). These animals were used for other studies as well. Surgery was carried out under deep anesthesia with Nembutal $(50 \mathrm{mg} / \mathrm{kg})$, followed by atro- 
pine sulphate $(0.5 \mathrm{ml} 0.1 \%$ solution $)$, intramuscularly, using sterile technique. The femoral vein was cannulated and bilateral sympaticotomy performed. A headset was implanted in the frontal bone. A small craniotomy (5 $\mathrm{mm}$ ) and duratomy was performed over the central projection zone of area 17 near the border with area 18. A precise histological reconstruction of the individual electrode tracks was not done. We were confident that we recorded from neurons in cortical areas 17 and 18 on the basis of localization of the trepanation site, the depth of microelectrode position below the cortical surface and the characteristics of the RFs.

During the experiment, the cat was fixed in an experimental instrument by the headset and lightly anaesthetized with a mixture of nitrous oxide and oxygen (70:30). The animal was paralyzed with a permanent intravenous infusion of muscle-relaxant tubocurarine chloride 1.2 $\mathrm{mg} / \mathrm{kg}$ per hour throughout the experiment. All the wounds were infiltrated with long lasting local anesthetics. Corneas were protected by neutral soft contact lenses and refractive errors corrected by spectacle lenses. The cat looked at a stimulus presented on the screen at a distance of $144 \mathrm{~cm}$. All the stimulation was monocular. After the investigation was completed, the animal was sacrified by overdose of Nembutal. Protocols and methods corresponded to the requirements of the Lithuanian Commission on Ethics for Studies involving Laboratory Animals.

Optical stimulation was carried out on the mechanically and optically supplemented two-canal automatic projector equipped with the electrical shutters, rotating optical prisms on step drivers and movable mirrors driven by personal computer. A light spot $(0.4 \mathrm{deg}$ in diameter) or a system of two such spots moving over the semi-translucent tangent screen was used for stimulation. Background luminance was $0.15 \mathrm{~cd} / \mathrm{m}^{2}$.

Extra cellular recordings were made from single cells in visual cortex by electrolytically sharpened varnished tungsten microelectrodes. An automatic driver advanced the microelectrode in minimum step of 1 micrometer with depth monitoring by a special electronic table. Signals were amplified, sent to window discriminator and monitored through loudspeaker and oscilloscope. The isolated spikes were stored like sequence of codes to indicate the time of occurrence (1 ms resolution). The boundaries of the RF and light active RF centre for each DS cell encountered were mapped on the screen with a flashing and moving light spot of the hand held electric ophthalmoscope. The location of area centralis was derived from the position of the optic disk, plotted on the screen by the mean angular separation [17].

The experiment consisted of two parts.

Firstly the directional characteristics of neurons were registered. The stimulus (single light spot) moved across the centre of the RF in 12 directions with step of $30^{\circ}$ (in the range from 0 to $360^{\circ}$ ), in randomized order. The amplitude of motion always substantially (by $40 \%$ ) exceeded the RF width. Optimal speed of the spot movement and spot luminance were chosen for each neuron. A post stimulus time histogram PSTH (10-20 consecutive presentations; bin width $20 \mathrm{~ms}$ ) was compiled in response to a moving light spot in the single direction (Figure 1(a)). From these 12 PSTHs the directional-tuning diagram was formed (Figure 1(b)). The directional-tuning bandwidth was calculated as the full width of the tuning curve at 0.63 of its maximum height. The PD was calculated by the method of linear approximation with reference to the value of weights in the adjacent directions. We concerned as DS neurons those cells that had the directional index (DI) higher than 0.5. DI was defined as a relative difference in responses to a preferred direction of movement $\left(\mathrm{R}_{\mathrm{pref}}\right)$ and opposite to it.

$$
\mathrm{DI}=\left(\mathrm{R}_{\text {pref }}-\mathrm{R}_{\text {backgr }}\right) /\left(\mathrm{R}_{\text {pref }}\right)
$$

where $R_{\text {pref }}$ and $R_{\text {backgr }}$ expressed as maximum firing rates in indicated direction minus the maximum rate of background activity.

Further investigation was performed only on DS neurons, which rather well responded to moving light spot.

Secondly, we investigated how the PDs of the DS neurons depend on the angle between the two moving spots. The angle was changed with a step of $30^{\circ}$ ranging from 0 to $180^{\circ}$. Two identical light spots of equal luminance and at the same speed moved simultaneously with

a) b)

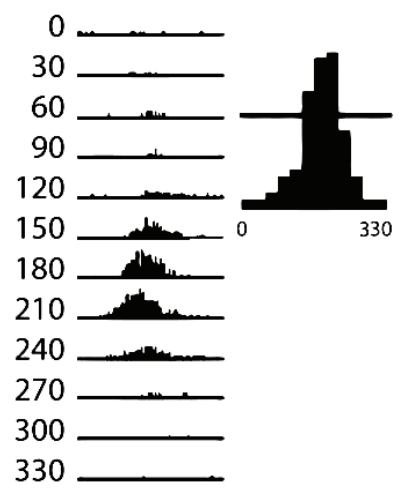

Figure 1. (a) - Post-stimulus-time histograms of responses to a light spot stimulus moving across the RF of a simple cell in an indicated direction. Bin width $20 \mathrm{~ms}, 20$ consecutive presentations, velocity $-2 \mathrm{deg} / \mathrm{s}$; (b) - Directional tuning diagram. Maximal rate of response $-45 \mathrm{imp} / \mathrm{s}, \mathrm{PD}-220^{\circ}$, directional-tuning bandwidth $-70^{\circ}$. 
the tested angle between their directions and with an intersection in the centre of RF (Figure 2(c)). The movement direction of the test spot was as a reference for two spots' motions. The test spot moved over the centre of the RF in 12 directions with the step of $30^{\circ}$ in a randomized order. The movement direction of the conditioning spot was shifted counterclockwise by the tested angular separation from the test spot movement direction. PSTHs of responses to a pair of moving light spots were compiled (Figure 2(a)), and the directional tuning diagram was formed (Figure 2(b)).

Influence of the relative luminance of two moving stimuli. The investigations were performed on twenty adult cats (weight $2.2-3.5 \mathrm{~kg}$ ).

The experiment consisted of a sequence of different procedures.

The method for estimation of the directional characteristics of neurons under presentation of a single spot has been already described.

The velocity sensitivity of the neurons was determineed. The response of the DS neuron to the single light spot moving in PD across the RF with different speed of the movement was registered. The tested velocities ranged from 2 to $80 \mathrm{deg} / \mathrm{s}$. The characteristic was made up from maximum values taken from corresponding PSTHs, compiled with a $50 \mathrm{~ms}$ bin width.

The contrast sensitivity of the neurons was determined.

a) b)

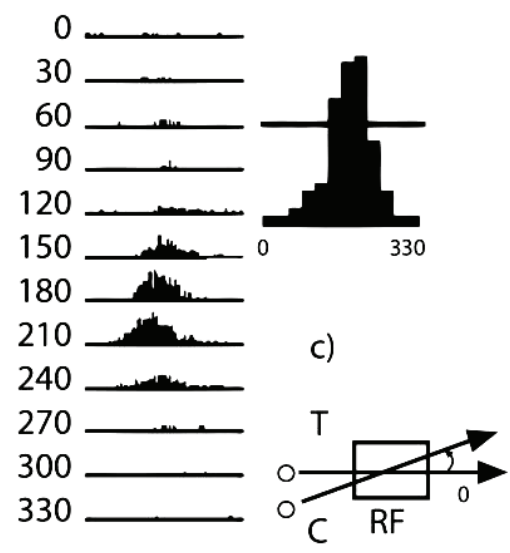

Figure 2. (a) - Post-stimulus-time histograms of response to a two light spot stimuli moving across of RF of the same simple cell in an indicated direction for test stimulus $(\mathrm{T})$. The moving direction of conditioning stimulus differed by the angle of $30^{\circ}$. Bin width $20 \mathrm{~ms}, 20$ consecutive pr- esentations, velocity $-2 \mathrm{deg} / \mathrm{s}$; (b) - Directional tuning diagram. Maximal rate of response -47 $\mathrm{imp} / \mathrm{s}$. PD $-200^{\circ}$, directional-tuning $-90^{\circ}$; (c) - Situation of stimulation.
Luminance contrast of a stimulus is determined by a ratio of the difference between the highest and the lowest luminance value to their sum in percentage terms:

$$
\mathrm{Lc}=(\mathrm{Lmax}-\mathrm{Lmin}) /(\mathrm{Lmax}+\mathrm{Lmin}) \times 100
$$

A change in contrast can be obtained modifying the spot luminance. For this purpose, the response of the DS neuron to a single light spot moving in PD across the RF was registered. The speed of spot movement was selected in the velocity response curve. The luminance of the spot varied from 0.001 to $5 \mathrm{~cd} / \mathrm{m}^{2}$ by neutral density filters. Background activity was subtracted from the response. The spot luminance was expressed in $\mathrm{cd} / \mathrm{m}^{2}$ as $\mathrm{L}_{\max }-\mathrm{L}_{\mathrm{bkg}}$.

We investigated how the PD of DS cells depend on relative luminance of two light spots moving in the cell's RF. Two light spots moved simultaneously with the angle of $60^{\circ}$ between their trajectories and intersected in the centre of RF. The test spot speed and luminance were selected from velocity and contrast response curves (Figures 5 and 6) correspondingly. The single test spot was moved across the RF in 12 directions with the step of $30^{\circ}$ in a randomized order and the preferred direction was specified. The preferred movement direction of the test spot for the given neuron changed while the additional spot (conditioning stimulus) was presented. The changes of the PD were measured according to the test spot (reference stimulus). The movement direction of the conditioning spot was shifted counter-clockwise from the direction of the test spot movement. The luminance of the conditioning spot was altered by neutral density filters in the range from 0.001 to $5 \mathrm{~cd} / \mathrm{m}^{2}$.

The evaluations of neuron response to a single spot and pair of stimuli were performed by nonparametric Wilcoxon rank method.

\section{RESULTS}

Influence of the angular separation between stimuli. The investigation was performed on 23 DS neurons: 11 simple and 12 complex.

The neurons were classified as simple or complex using conventional criteria $[18,19]$, such as sensitivity to stimulus speed, the size of receptive-field, the level of spontaneous activity and spatial arrangement of light excitatory and inhibitory zones.

Since no principal difference among these neurons was observed in the data of this investigation, the results were combined. All the cells had the RF within $15 \mathrm{deg}$ of the centre of the area centralis in lower half of the contralateral visual field.

The DI for all cells was from 0.6 to 1.5 , mean 0.9 . Directional-tuning bandwidth for spot stimulus was from 60 to $210^{\circ}$, mean $90^{\circ}$. 
We investigated the dependence of the PD of DS neurons on the angle between the directions of the movement of the two spots. The PD was calculated from directional tuning diagram at each angle (see Figure 2(b)). The dependence shows that the increase of the angle between the directions of the two moving spots shifts the PD clockwise (Figure 3).

When the angle between the directions of the two moving spots was more than $60^{\circ}$, two peaks on the DS tunes diagrams were obtained. For different neurons, the second PD appears at different angles, such as 90, 120 or $150^{\circ}$.

The analysis of the responses of the neurons shows that at great angular separation two PDs obtained in two spots' experiments approach a neuron's PD obtained for one of the spots (one for test spot $(\mathrm{T})$ and one for conditioning spot (C) in Figure 3.

The single PD was as usually seen when the angle between the directions of the two moving spots was 30 or $60^{\circ}$. The directional tuning bandwidth in these situations was usually broader than for a single spot. The PD in most cases was shifted clockwise, but this shift was different for different neurons. Figure 4 shows the distribution of the shift of the PD for 18 neurons. Data analysis showed that when two spots moved width the angle of $30^{\circ}$ between their trajectories, 11 out of 18 neurons $(61 \%)$ had the shift of the PD close to the bisector, 2 out $18(11 \%)$ had the shift less than $10^{\circ}$, and 5 of 18 (28\%) had the shift more than $20^{\circ}$. When the angle between the trajectories of the two moving spots was $60^{\circ}$, 12 out of 18 neurons $(66 \%)$ had the shift of the PD close to the bisector, 1 out 18 neurons $(6 \%)$ had the shift less than $20^{\circ}$, and 5 out $18(28 \%)$ had the shift more than $40^{\circ}$.

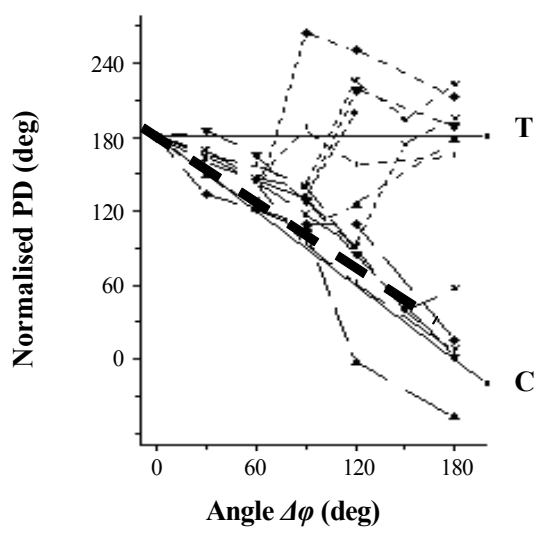

Figure 3. Dependence of the $\mathrm{PD}$ on the angle between the two moving spots for eighteen neurons: $\mathrm{T}$ - the level of the response to test spot alone, $\mathrm{C}$ - for conditioning spot. The averaged shift of the set of neurons PD is marked by the solid dashed line.
The PD was close to the bisector at both angles (30 and $\left.60^{\circ}\right)$ for 10 out of 18 neurons $(56 \%)$. The non-parametric statistics showed the reliable difference of PD shift obtained at angles of 30 and $60^{\circ}$, but do not support the grouping of neurons by value of the shift.

Influence of the relative luminance of two moving stimuli. The investigation was performed on 24 DS neurons: 10 simple and 14 complex.

No significant differences were observed between these types of neurons in respect of the investigation presented. All the cells had the RF within $15 \mathrm{deg}$ of the centre of the area centralis in the lower half of the contralateral visual field.

It was discovered that different movement speed was optimal for certain number of DS neurons in this population (see Figure 5). The velocity of $2 \mathrm{deg} / \mathrm{s}$ was optimal for 2 cells $(8 \%), 5 \mathrm{deg} / \mathrm{s}$ - for 4 cells $(17 \%), 10 \mathrm{deg} / \mathrm{s}-$ for 9 cells $(38 \%), 20 \mathrm{deg} / \mathrm{s}-$ for 4 cells $(17 \%), 40 \mathrm{deg} / \mathrm{s}$ - for 4 cells (17\%) and $80 \mathrm{deg} / \mathrm{s}$ - for 1 cell (4\%).

In the experiment with two stimuli we investigated how the PD of DS neurons depend on the relative luminance of the conditioning spot. The angle between the two moving spots was $60^{\circ}$. The speeds of both moving

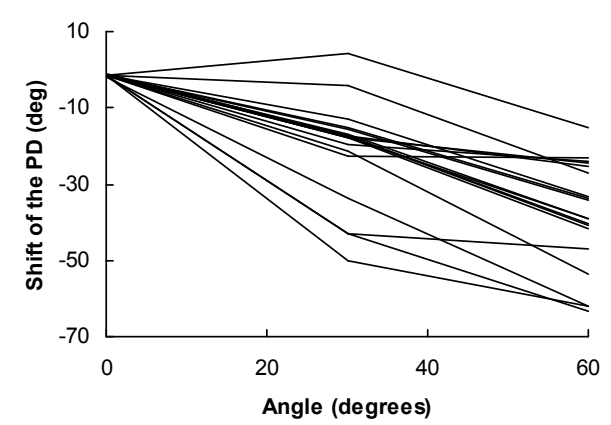

Figure 4. Distribution of the shift of the PD for eighteen neurons in dependence on the angle between two moving spots.

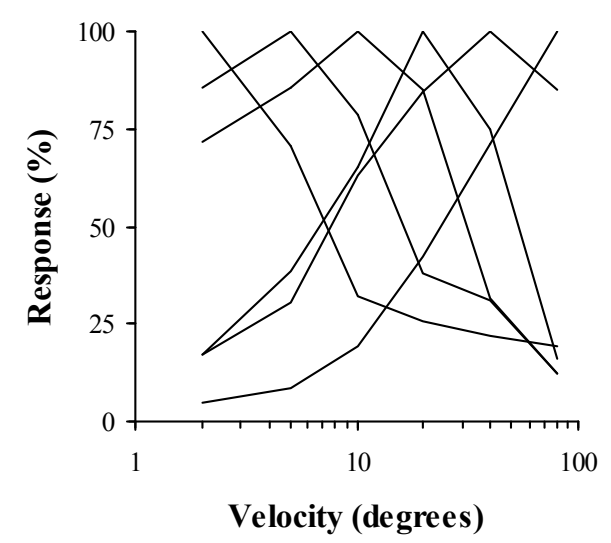

Figure 5. The response dependence on moving spot velocity for six types of neurons. 
spots were equal and were optimal for the given neurons (specified from velocity responses curves (Figure 5)). The value of test spot luminance was chosen at a $50 \%$ response level using a stimulus-luminance curve (see Figure 6). In Figure 7 the dependence of the shift of the $\mathrm{PD}$ on the relative luminance of the conditioning spot is presented. On the y axis there is expressed the difference of the PD obtained when luminance of conditioning spot was equal to test spot luminance, and when the luminance of the conditioning spot was different. The negative values on the $y$ axis correspond to the shift of the PD counterclockwise. The positive values on the $y$ axis correspond to the shift of the PD clockwise, i.e., an increase of the initial shift. On the $\mathrm{x}$ axis there is expressed the ratio of the luminance of the conditioning spot to test spot luminance.

The non-parametric statistics showed the reliable difference of PD shift obtained in situations when the relative luminance of conditioning spot was more than test

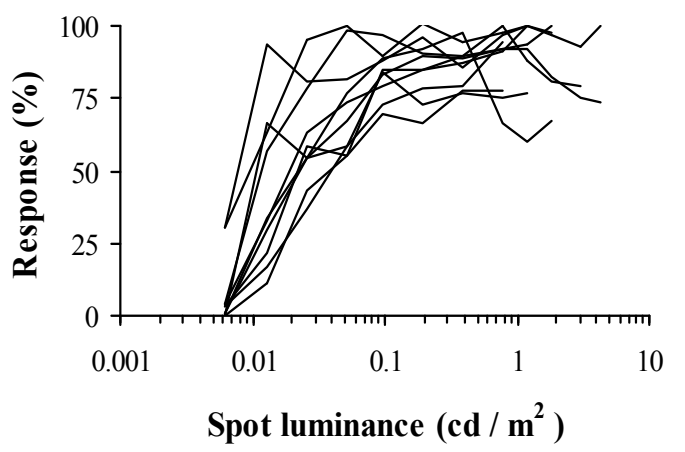

Figure 6. Representative response dependence on stimulus luminance for ten neurons.

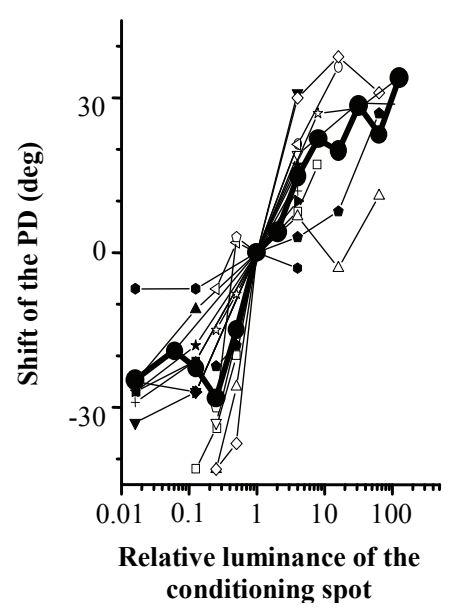

Figure 7. The dependence of the shift of the $\mathrm{PD}$ on the relative luminance of the conditioning spot for twenty neurons. The averaged shift of the set of neurons PD is marked by the solid line. spot, and when conditioning spot luminance was lower than test spot luminance.

\section{DISCUSSION}

Understanding of motion perception requires an ascertaining of coding features of motion parameters in visual cortex.

We investigated the population of DS cells in the primary visual cortex. These cells differ by the width of the DS tuning diagram: from sharp selective to broad selective. DS cells can deal width different tasks of motion processing. Some of them can act as motion detectors, others as motion predetectors (the broad selective ones). Our investigation has revealed two ranges of the angles between two moving stimuli, which evoked different interaction of RF subunits in DS cells:

When the angle between the directions of the two moving spots was 30 or $60^{\circ}$, the PD for $56 \%$ of cells was close to the bisector. These cells responded to the combination of the two moving stimuli exactly as to single stimulus moving in an intermediate direction (this direction coincided with PD of the DS neuron to a single spot). This suggests that these DS cells can linearly sum the output signals of predetectors.

When the angle between the directions of the two moving spots exceeded $60^{\circ}$, two peaks on the DS tuned diagram were obtained. Each of them was evoked by one of the two moving stimuli and corresponded to the same PD. It means that in this case the output signals of the DS cells are determined by single output of the predatector. In other words, such DS cells act as detectors, which "see" only one (the right direction) stimulus of the two.

This corresponds to the bimodal directional tuning, obtained as a separate response of neuron to the motion of the two component gratings as referred to in the investigations of striate cortex. Also, our investigation reveals that DS neurons respond to moving stimuli at an angle of $180^{\circ}$ (to stimuli moving in preferred and opposite directions simultaneously). This indicates that such motion detection models, where signals from two motion detectors to opposite directions are subtracted, are unsuitable for DS neurons in cat's striate cortex [20,21].

So, these relationship show that when the luminance of the conditioning spot is lower than the luminance of the test spot and decrease, the shift of the PD decrease too approaches the value of the PD for test stimulus alone. While the luminance of the conditioning spot exceeds the luminance of the test spot, then the shift of the PD increases. When the luminance of conditioning spot is higher than the luminance of the test spot, and it increases then the shift of the PD increases too. 
Thus, alteration of the relative luminance of two moving spots by changing the luminance of the conditioning spot evokes the deviation of the PD of DS neurons towards the direction of the more intensive stimulus in pair. Similar results were obtained in psychophysical experiments on human subjects with apparent motion or with random dot stimuli [22]. Such familiarity can indicate that some features of basic principles that are necessary for discrimination and perception of motion direction are already presented at the level of striate cortex.

\section{CONCLUSIONS}

1) When the two stimuli moving in different directions (angles between movement directions were $30^{\circ}$ and $60^{\circ}$ ) are projected in the receptive field of directional sensitive neurons, the preferred direction for $56 \%$ of tested neurons was close to the bisector of angles mentioned above. Thus, these neurons selective to movement direction averaged the movement directions of two moving bright spots.

2) When the relative luminosity of two spots moving in different directions was changed, the preferred direction of the DS neurons was also changed. The preferred direction was shifted toward the brighter spot. The greater was the difference in luminosity of two spot the larger was the shift of the preferred direction.

\section{REFERENCES}

[1] Cleland, B.G. and Levick, W.R. (1974) Properties of rarely encountered types of ganglion cells in the cat's retina and an overall classification. Journal of Physiology, 240(2), 457-492.

[2] Stone, J. and Fukuda, Y. (1974) Properties of cat retinal ganglion cells: a comparison of W-cells with $\mathrm{X}$ - and Y-cells. Journal of Neurophysiology, 37, 722-748.

[3] Hoffmann, K.P. (1973) Conduction velocity in pathways from retina to superior colliculus in the cat: A correlation with receptive-field properties. Journal of Neurophysiology, 36(3), 409-424.

[4] Hoffmann, K.P. and Stone, J. (1985) Retinal input to the nucleus of the optic tract of the cat assessed by antidromic activation of ganglion cells. Experimental Brain Research, 59(2), 395-403.

[5] Cleland, B.G., Levick, W.R., Morstyn, R. and Wagner, H.G. (1976) Lateral geniculate relay of slowly conducting retinal afferents to cat visual cortex. Journal of Physiology, 255(1), 299-320.

[6] Stone, J. and Dreher, B. (1973) Projection of X- and Ycells of the cat's lateral geniculate nucleus to areas 17 and 18 of visual cortex. Journal of Neurophysiology, 36(3), 551-567.

[7] Orban, G.A. and Callens, M. (1977) Receptive field types of area 18 neurones in the cat. Experimental Brain Research, 30(1), 107-123.

[8] Orban, G.A., Kenedy, H. and Maes, H. (1981) Response to movement of neurons in areas 17 and 18 of the cat: Direction selectivity. Journal of Neurophysiology, 45(6), 1059-1073.

[9] Tretter, F., Cynader, M. and Singer, W. (1975) Cat parastriate cortex: A primary or secondary visual area. Journal of Neurophysiology, 38(5), 109-113.

[10] Movshon, J.A., Thompson, I.D. and Tolhurst, D.J. (1978) Receptive field organization of complex cells in the cat's striate cortex. Journal of Physiology, 283, 79-99.

[11] Emerson, R.C., Bergen, J.R. and Adelson, E.H. (1992) Directionally selective complex cells and the computation of motion energy in cat visual cortex. Vision Research, 32(2), 203-218.

[12] Hammond, P. and MacKay, D.M. (1975) Differential responses of cat visual cortical cells to textured stimuli. Experimental Brain Research, 22(4), 427-430.

[13] Jagadeesh, B., Wheat, H.S. and Ferster, D. (1993) Linearity of summation of synaptic potentials underlying direction selectivity in simple cells of the cat visual cortex. Science, 262(5141), 1901-1904.

[14] Emerson, R.C. (1997) Quadrature subunits in directionally selective simple cells: Spatiotemporal interactions. Visual Neuroscience, 14(2), 357-371.

[15] Barlow, H.B. and Levick, W.R. (1965) The mechanism of directionally selective units in rabbit's retina. Journal of Physiology, 178(3), 477-504.

[16] Orban, G.A., Kenedy, H. and Maes, H. (1981) Response to movement of neurons in areas 17 and 18 of the cat: direction selectivity. Journal of Neurophysiology, 45(6), 1059-1073.

[17] Bishop, P.O., Kozak, W. and Vakkur, G.J. (1962) Some quantitative aspects of the cat's eye: Axis and plane of reference, visual field co-ordinates and optics. Journal of Physiology, 163(3), 466-502.

[18] Hubel, D.H. and Wiesel, T.N. (1962) Receptive fields, binocular interaction and functional architecture in the cat's visual cortex. Journal of Physiology, 160(1), 106154.

[19] Henry, G.H. (1977) Receptive field classes of cells in the striate cortex of the cat. Brain Research, 133(1), 1-28.

[20] Adelson, E.H. and Berger, J.R. (1985) Spatiotemporal energy models for the perception of motion. Journal of the Optical Society of America A, 2(2), 284-291.

[21] Van Santen, J.P. and Sperling, G. (1985) Elaborated Reichardt detectors. Journal of the Optical Society of America A, 2(2), 300-321.

[22] Zohary, E., Scase, M.O. and Bradic, O.J. (1996) Integration across directions in dynamic random dot displays: vector summation or winner take all? Vision Research, 36(15), 2321-2331. 